\title{
Energy Efficient Building Environment Control Strategies Using Real-time Occupancy Measurements
}

\author{
Varick L. Erickson ${ }^{1}$, Yiqing Lin ${ }^{2}$, Ankur Kamthe ${ }^{1}$,Rohini Brahme ${ }^{2}$, \\ Amit Surana ${ }^{2}$, Alberto E. Cerpa ${ }^{1}$, Michael D. Sohn ${ }^{3}$ and Satish Narayanan ${ }^{2}$ \\ ${ }^{1}$ University of California - Merced \{verickson, akamthe,acerpa@ucmerced.edu\} \\ ${ }^{2}$ United Technologies Research Center \{LinY,BrahmeR,SuranaA,NarayaS@utrc.utc.com $\}$ \\ ${ }^{3}$ Lawrence Berkeley National Laboratory \{MDSohn@lbl.gov\}
}

\begin{abstract}
Current climate control systems often rely on building regulation maximum occupancy numbers for maintaining proper temperatures. However, in many situations, there are rooms that are used infrequently, and may be heated or cooled needlessly. Having knowledge regarding occupancy and being able to accurately predict usage patterns may allow significant energy-savings by intelligent control of the L-HVAC systems. In this paper, we report on the deployment of a wireless camera sensor network for collecting data regarding occupancy in a large multi-function building. The system estimates occupancy with an accuracy of $80 \%$. Using data collected from this system, we construct multivariate Gaussian and agent based models for predicting user mobility patterns in buildings. Using these models, we can predict room usage thereby enabling us to control the HVAC systems in an adaptive manner. Our simulations indicate a $14 \%$ reduction in HVAC energy usage by having an optimal control strategy based on occupancy estimates and usage patterns.
\end{abstract}

\section{Introduction}

Heating ventilating and air conditioning (HVAC) systems account for 50\% of the total energy budget in buildings [5]. Prior research has shown that energy savings are achievable by regulating fresh air ventilation based on the total number of occupants in a building. This procedure is referred to as demand-controlled ventilation, and studies suggest 10 to $15 \%$ of HVAC energy can be reduced in buildings that set ventilation rates based on maximum occupancy [2].

In general, the approach used is to assume that all rooms are occupied during working hours and not being used during the night. However, it is obvious that this does not maximize energy savings. Rooms are often left empty during part of the day or perhaps are only used semi regularly, e.g. con-

Permission to make digital or hard copies of all or part of this work for personal or classroom use is granted without fee provided that copies are not made or distributed for profit or commercial advantage and that copies bear this notice and the full citation on the first page. To copy otherwise, to republish, to post on servers or to redistribute to lists, requires prior specific permission and/or a fee. ference rooms. It would be more efficient to only condition rooms during the times that are actually occupied. Using an L-HVAC system (lighting, heating, venting, and air conditioning system), various environmental aspects of room can be controlled for energy savings. Thus, knowledge of occupancy is crucial in order to maximize efficiency of a system.

If room occupancy is known, then specific strategies can be used to condition the room optimally. The lack of reliable data (both real time and model) for the movement of people inside buildings may make many of these aspects difficult to control efficiently. Although many modern buildings include motion detectors and temperature and $\mathrm{CO}_{2}$ sensors for light and air flow management, these sensors present some limitations. Motion detectors provide an efficient way to detect occupancy, but they provide no information about the quantity of people using the space since their output is a binary process; actual occupancy is required to optimally control $\mathrm{CO}_{2}$ levels. While temperature and $\mathrm{CO}_{2}$ sensors may provide better indirect measures of actual occupancy, the physical phenomena being measured responds to different time scales and these sensors may be more suitable for understanding general trends at large time scales. A faster new distributed sensor network may be needed to respond quickly to ever changing occupancy. Therefore, conditioning of the room must begin prior to when the room is actually utilized. Thus, having the capability to predict user movement or room usage patterns prove to be helpful for demand-control ventilation; they may also provide enough information for even substantial local-scale controls. Occupancy prediction can be achieved by modeling long traces of occupancy data captured by a sensing system [6], helping modify L-HVAC control strategies as building usage patterns change with time.

In this paper, we describe the experiments conducted with SCOPES [4], a wireless camera sensor network for gathering traces of human mobility patterns in buildings. With this data and knowledge of the building floorplan, we created two prediction models for describing occupancy and movement behavior. The first model comprises of fitting a Multivariate Gaussian distribution to the sensed data and using it to predict mobility patterns for the environment in which the data was collected. The second model is an Agent Based Model (ABM) that can be used for simulating mobility patterns for developing HVAC control strategies for buildings that lack an occupancy sensing infrastructure. We integrate the sim- 
ulation information to compute energy saving for a building where we can adjust L-HVAC controls based on occupancy estimates from a sensor network.

The paper is organized as follows: Section 2 describes the wireless camera sensor network used for occupancy estimation. Section 3 describes how occupancy data is used to develop models for user mobility prediction and energy saving estimation and Section 4 compares the performance of these models. Section 5 discusses the L-HVAC control strategies and energy savings. Section 6 summarizes our paper and discusses future work.

\section{Occupancy Sensing}

\subsection{Hardware and Software Infrastructure}

We deployed the SCOPES system [4] comprising of 16 sensor nodes on the ceiling of the corridors in the University of California - Merced Science and Engineering building. The nodes were deployed in groups of three at transition points of the floorplan. Each sensor node is comprised of a $\mathrm{Cy}$ clops camera interfaced with a Moteiv Tmote Sky module via an intermediate adapter board. The Cyclops is a very low power camera with an on-board $4 \mathrm{MHz}$ ATmega128L micro-controller (MCU) and 512KB of external SRAM. Due to the limitation of the total addressable memory, the external SRAM is divided into eight, 64KB memory banks. The Cyclops captures $1064 \times 64$ pixel grayscale images per bank (i.e., 80 total). In each group, multiple nodes sense the same area with coordination such that there is minimal overlap between the image capture periods of the cameras. Whenever a person crosses any one of these transition points, the cameras capture and process the image data and, thus indicating whether the transition was recorded or not.

Due to the severe limitations on the available computational power, we execute lightweight processing algorithms on the Cyclops. The object detection algorithm running on the Cyclops determines the presence of an object in the image foreground, if any, and updates the background. This is done using a modified background subtraction algorithm that labels the pixels in the captured images as object, shadow or background depending upon a preset threshold. Next, we use a connected components algorithm to merge all pixels labelled as object into a blob. For each blob, information regarding the centroid ( $\mathrm{x}$ and $\mathrm{y}$ coordinates) and the number of pixels is recorded. We track the movement of the blob across images based on displacement with respect to the previous image. After processing all the images in the current bank, an array of data structures containing information on a maximum of four objects per bank is transferred to the Tmote using serial communication. The Tmote routes the information to the base station using multihop communication. Both the Cyclops and the Tmote run the TinyOS operating system.

\subsection{System Evaluation and Data Verification}

We collected data using the SCOPES system for a period of 24 hours. This was done to evaluate the performance of the system with respect to the ground truth. Overall, the SCOPES system detected $80 \%$ of all recorded transitions, as compared to the ground truth, over a period of 24 hours. The system has a false positive rate of $25 \%$, which was higher than the one encountered in the 2-hour experiments $(18.5 \%)$ conducted in [4]. The false positive rate refers to the ratio of number of transitions detected by SCOPES when the ground truth shows none to the total number of transitions detected by SCOPES. False positives result mainly, due to the fixed thresholds in the background subtraction algorithms and camera hardware calibration.

For collecting the ground truth to compare the performance of SCOPES, we installed three Philips SPC-900NC web cameras to record the movement of people. The camera captured approximately three images every two seconds. This frequency is high enough to record transitions across areas of interest to the accuracy of seconds. We collected data from the ground truth system for the entire 24 hours that the SCOPES system was operated and then for a further 24 hours. The ground truth data was processed using Perlmagick which annotates images with people in them. We manually corrected the Perlmagick output for the false positives and false negatives in the processed ground truth. Our goal was to use the SCOPES dataset to characterize the sensor error and the ground truth data for creating models of user mobility patterns.

From the ground truth data, we defined transition points in the floorplan (refer Figure 1). The time, direction, and number of people transitioning were recorded for each transition. The sign of the transition is used to indicate direction of travel. The transitions points are strategically placed at entrances/exits to capture the occupancy changes of each area.

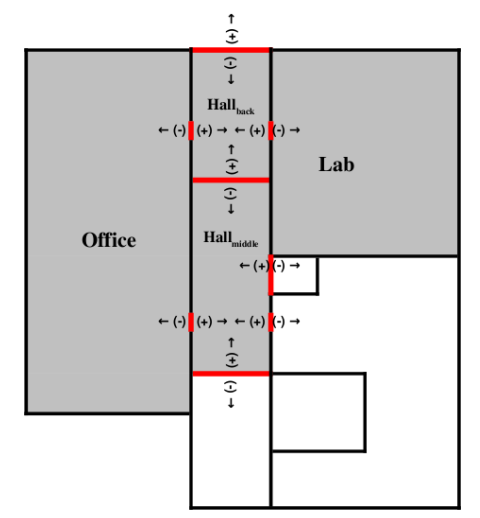

(a) Occupancy Areas

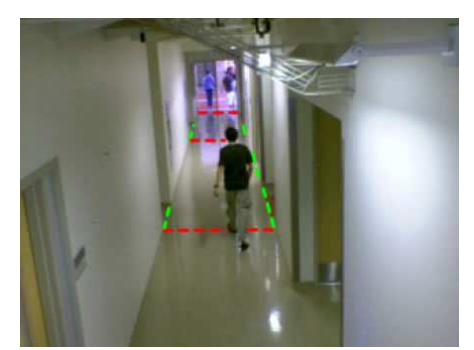

(b) Sample Camera Image

Figure 1. The red and green lines indicate the locations of the transition boundaries. The arrows show the sign associated with each transition direction. The gray areas indicate the area occupancies that can be derived from the transition data. 


\section{Occupancy Models}

Understanding the dynamics of occupancy patterns is central to the approach of occupancy-based building energy management. Multi-scale spatiotemporal dynamics of occupancy with high variability makes this a challenging task. In this paper, we developed two dynamic models of occupancy distributions, the Multivariate Gaussian Model and the Agent-Based simulation Model (ABM). These models enable simulations at an individual level and are useful for off-line studies, such as: 1) learning occupancy patterns from sensor data and generating variations of those patterns, which can be used for evaluating different energy management options, 2) optimal sensor placement for accurate estimation of occupant traffic, and 3) learning statistical patterns in occupant traffic in one building and applying it to other buildings of similar types to simulate occupancy dynamics.

\subsection{Multivariate Gaussian Model}

In this section we discuss a simple occupancy model that utilizes multiple gaussian fits over the data. In particular, we focus on constructing a model that can simulate the occupancy for two specific areas that are represented by the ground truth data. Given its simplicity, this model serves as a coarse baseline model for other occupancy simulation and prediction approaches.

\subsubsection{Training}

On an intuitive level, over the course of a day we expect occupancy to increase in the morning when people arrive for work, decrease when people go to lunch, increase when people return from lunch, and then eventually drop to zero when people leave for the day. These are the general increases and decreases of occupancy we can expect based on our real world experiences. The strategy behind this approach is to model each of the increases and decreases separately.

However, as the occupancy data suggests in Figure 2, there may be other regular phenomenon affecting occupancy other than those based on intuition. In order to ensure that the majority of occupancy dynamics is captured, the data is partitioned into hourly blocks. Let $O_{h}$ denote all occupancies that occur per second during hour $h$ where $1 \leq h \leq 24$,

$$
O_{h}=\left\{\begin{array}{cccc}
H B_{1} & H M_{1} & L_{1} & O f_{1} \\
\vdots & \vdots & \vdots & \vdots \\
H B_{n} & H M_{n} & L_{n} & O f_{n}
\end{array}\right\}
$$

$H B, H M, L$, and $O f$ refers to the Hallway ${ }_{b a c k}$, Hallway middle $_{\text {, }}$ Lab, and Office areas occupancies respectively (refer Figure 1). $n$ represents the number of observations in hour $h$. Let $\mu_{H B}, \mu_{H M}, \mu_{L}$, and $\mu_{O f}$ denote the average occupancy for

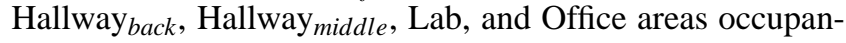
cies respectively. These means will change based on $h$.

We calculate a vector of means $\mu_{h}=\left\{\mu_{H B}, \mu_{H M}, \mu_{L}, \mu_{O f}\right\}$ and covariance matrix $\Sigma_{h}$ from $O_{h}$. Using $\mu_{h}$ and $\Sigma_{h}$, we define a probability density function (PDF) $f$ :

$f\left(O_{h} ; \mu_{h}, \Sigma_{h}\right)=\frac{1}{(2 \pi)^{\frac{n}{2}}\left|\Sigma_{h}\right|^{\frac{1}{2}}} \exp \left\{\frac{1}{2}\left(O_{h}-\mu_{h}\right)^{\prime} \Sigma_{h}^{-1}\left(O_{h}-\mu_{h}\right)\right\}$

The PDF $f$ allows us to describe the probability of an occupancy occuring for a specific hour dataset $O_{h}$. Using this function we can randomly draw occupancy vectors from the distribution. For hours 8:00:00 to 22:00:00, we use $f$ to describe the distribution of the occupancies. These are the hours that have the most occupancy dynamics. For hours 23:00:00 to 7:00:00, we assume that the occupancy for all areas is zero. This assumption is based on our observation of the 48 hours ground truth and intuition.

\subsubsection{Simulation Generation}

However, randomly drawing occupancies from $X_{h}$ does not represent certain constraints. For example, suppose we randomly sample from the distribution and obtain $\left\{\begin{array}{llll}0 & 0 & 0 & 0\end{array}\right\}$ for time $t$ (all areas are empty) and $\left\{\begin{array}{llll}0 & 0 & 0 & 1\end{array}\right\}$ for $t+1$ (one person in the office). This situation is impossible since a person must first pass through the hallway to reach the office. Thus, if we sample randomly from the fit distributions without restrictions, the simulation will produce impossible transitions.

In order to solve this problem, rather than sample from the entire distribution, we instead randomly sample from a subset of occupancies that are actually possible given the current occupancy state. The following algorithm is used to generate simulated occupancies:

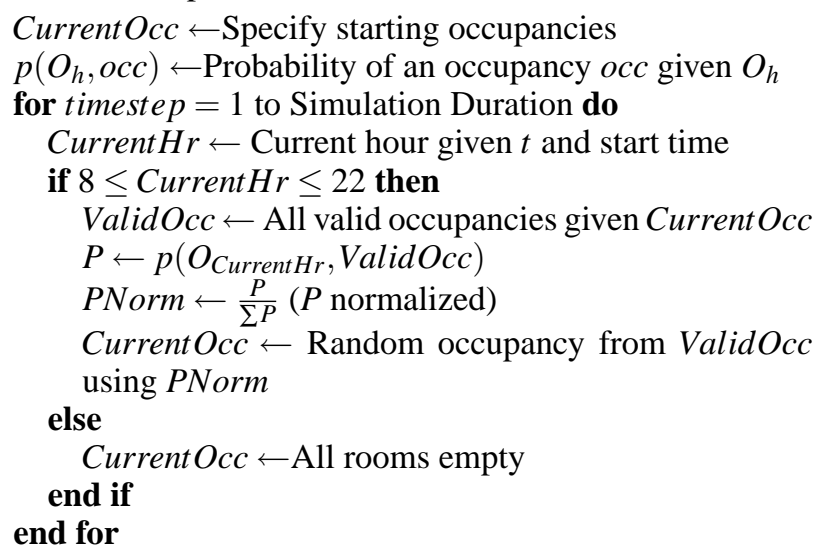

\subsubsection{Assumptions}

There are several assumptions that are made to increase efficiency and to account for physical restrictions. When determining valid occupancies, the list of possible occupancies is not exhaustive. We assume that a maximum of two people can move through the lab, office, and elevator doorways. For slightly larger hallway entrances, we assume a maximum of three people can pass over a transition boundary. These assumptions are based on the maximum transitions observed in the ground truth data. The last assumption made is that people are not moving through multiple doorways concurrently. This is done to reduce the total number of possible occupancies that need to be examined and the time required to run simulations. The data shows that transitions rarely occur concurrently. Out of the 48 hours of ground truth data, only $4.68 \%$ of transitions occurred concurrently.

\subsection{Agent Based Model}

An agent-based model of part of the 2nd floor of the Science and Engineering building at UC Merced has been developed to simulate people dynamics. The simulation model we have employed is analogous to the cellular automata models [7]. The model takes into account the building geometry and simulates each individual's movement, and is able to provide 

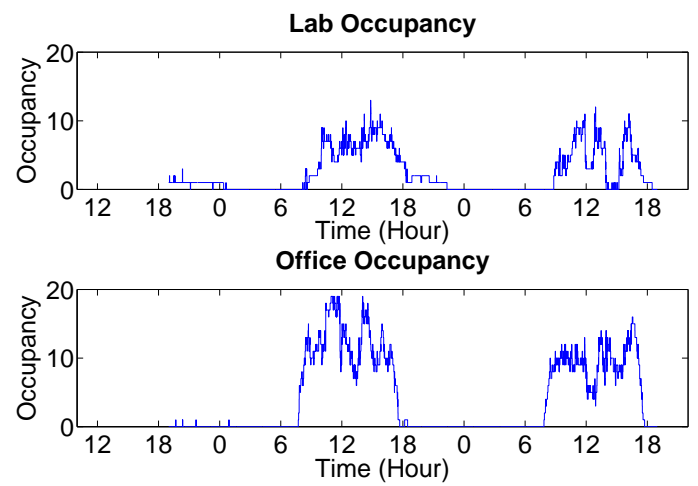

Figure 2. 48 hour ground truth occupancy data.

quantitative output such as occupancy and congestion levels at any location and at any time period. In order to simulate each individual's movement, agent decisions must be modeled on several levels, such as itinerary, path choice,and walking behavior [1]. Itinerary decision determines the time of arrival and entrance, the number of stops, and the location and dwell time of each stop, for each individual. Path choice is the decision on which path to follow, given the individual's destination and a set of alternatives. Walking behavior is determined by factors such as average speed, average space taken by each individual, and conflict resolution rules at times when conflicts between agents arise.

In our current implementation, the spatial grid size has been set to be 2 by 2 feet cell (with discrete time step of 0.5 sec) and an agent follows a shortest path. Occupant itinerary has been calibrated using arrival time and entrance, number of stops, and location and dwell time of each stop from traffic data collected from web cameras located along the corridors of the S\&E building. Preliminary results indicate that the calibrated model-based predictions are reasonable when compared to raw measurements. The calibration procedure is described as follows.

In the first step, individual camera data were combined based on camera location, direction and sensor event time stamps, converting raw measurements into meaningful trajectories. Each trajectory has a time stamp indicating its initiating time, and zone identification numbers indicating its start and end location in the building. For example, if the camera data indicates that boundary 1 has an event at time t, boundary 2 has an event at time $\mathrm{t}+\Delta$, and $\Delta$ is within the typical time range used to go from boundary 1 to boundary 2 , then a trajectory can be formed by combining the two events indicating at time $\mathrm{t}$, a person moves from zone $\mathrm{i}$ to zone $\mathrm{j}$ through boundary 1 and 2 . In the second step, a heuristic procedure was followed to assign trajectories to agents, from which agent itineraries were formed.

\section{Model Comparison}

In this section, we compare the performance of the MVGM and ABM, and examine the merits of each approach. The main statistics used for comparison are root mean squared error (RMSE) and normalized root mean squared error (NRMSE). The RMSE is the average difference in occu- pancy for a given simulation.

\subsection{Initial Exploration}

As expected, regular patterns in the occupancy data are observable (refer Figure 2). The office plot shows the occupancy steadily increasing until just after noon. At around 12:00:00, people in the office leave for lunch and then return shortly after. By 18:00:00, the entire office is empty. One noticeable discrepancy can be observed when comparing peak office occupancy of each day. The first day shows a maximum office occupancy of 18 whereas the second day shows a maximum occupancy of only 13 . This is due to a large lunch meeting of six people on the first day. Typically the office staff is around 12 people. The lab plot shows a slightly different patterns but still is consistent with intuition. Students arrive in the morning, go to lunch, and then stay until late evening. Unlike the office staff, many students arrive mid-afternoon and leave much later.

\subsection{Multivariate Gaussian Model Results}

The MVGM was trained using the first 24 hours of data from the ground truth. The second 24 hours of ground truth was used as a testing set. For each model, 20 simulations were generated. Figures 3 shows a sample simulation generated by this model. From the plots, we can see that the simulation seems to capture the major events that occur during the day such as arriving for work and going to lunch. One noticeable difference is that simulations contain a fair amount of noise. This is most likely caused by the random sampling. Though each occupancy sampled is possible under the sampling rules, the rules do not prevent certain types of unlikely events. For example, it is possible under our current scheme for a person to enter the hallway through a particular door and then with fairly high probability immediately exit out of the same door. This "pacing" behavior seems to be the cause of this noise. This could potentially be corrected with additional sampling rules. On average, simulated lab occupancy differs from the test set occupancy by 3.462 people (RMSE) which is an average error of $28.8 \%$ (NRMSE). The simulated office occupancy shows an average difference of 7.453 (NRMSE of $46.5 \%$ ). If we examine the combined total occupancy of both rooms, we find that the simulated total occupancy differs from the training set by 10.214 (NRMSE of $42.6 \%$ ).

\subsection{Agent Model Results}

The ABM was trained using the same 24 hour data set as the MVGM. To generate occupancy profile for lab and office, a day is divided into six time periods taking into account different behavior during different time of the day, e.g., early morning, lunch time and late afternoon. For each time period, empirical distributions of arrival time and duration in the lab/office were collected from the agent-based model. Based on the distributions, 20 simulation runs of the ABM were generated representing a variety of possible occupancy patterns of the lab and office. Figure 3 shows a sample simulation produced by the ABM. Like the MVGM, the ABM also seems to generate plausible simulations that capture the major shifts in occupancy. The simulated lab occupancy differs from the test set occupancy by 3.774 people (RMSE) 


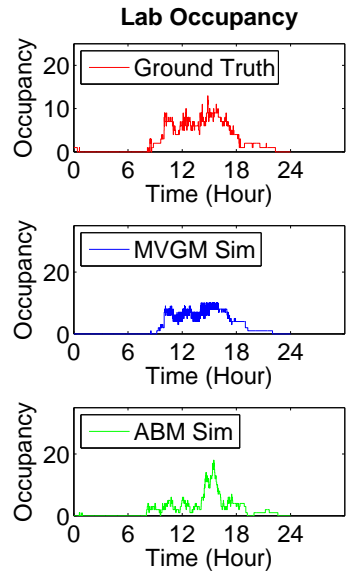

(a)
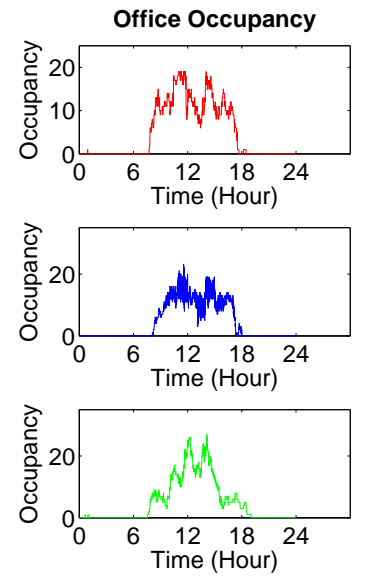

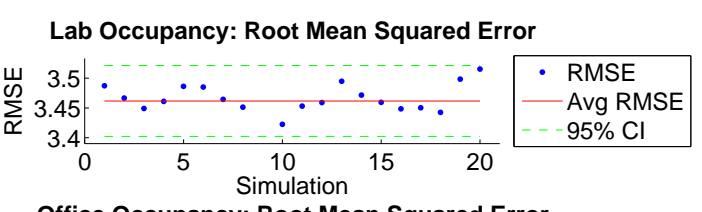

Office Occupancy: Root Mean Squared Error

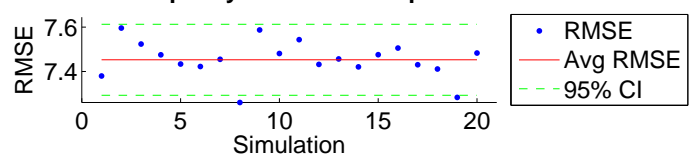

Total Occupancy: Root Mean Squared Error

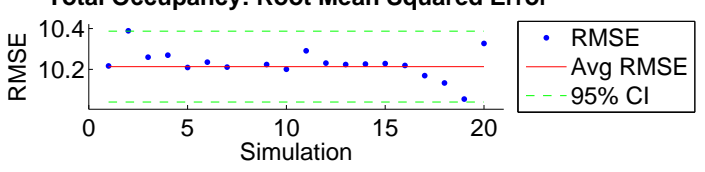

(b)

Figure 3. (a) Comparison of ground truth traces for lab and office areas with MVGM and ABM simulation traces. (b) Variation in RMSE along with $95 \%$ confidence interval for MVGM simulation traces.

\begin{tabular}{r|cc|cc} 
& \multicolumn{2}{|c}{ MVGM } & \multicolumn{2}{c}{ ABM } \\
& RMSE & NRMSE & RMSE & NRMSE \\
\hline Lab & 3.462 & 0.2885 & 3.774 & 0.3145 \\
Office & 7.453 & 0.4658 & 7.577 & 0.4736 \\
Total & 10.214 & 0.4256 & 10.395 & 0.4331
\end{tabular}

Table 1. This table shows the average RMSE and NRMSE for simulations generated by the MVGM and the ABM.

\begin{tabular}{r|cc} 
& RMSE & NRMSE \\
\hline Lab & 3.370 & $19.4 \%$ \\
Office & 3.107 & $28.1 \%$ \\
Total & 5.688 & $23.7 \%$
\end{tabular}

Table 2. This table shows the RMSE and NRMSE when comparing the 24 hour testing and training datasets.

which is an average error of $31.5 \%$ (NRMSE). The simulated office occupancy shows an average difference of 7.577 (NRMSE of 47.4\%). The simulated total occupancy differs from the testing set by 10.395 (NRMSE of 43.3\%).

\subsection{Comparison of Models}

With respect to RMSE and NRMSE, both models to have similar performance. Though both models have a large amount of error, this error is actually reflecting the amount of occupancy variation that is possible between different days. If we compare the 24 hours testing set to the 24 hours training set, we find that the NRMSE for the lab, office and total occupancies are $28.1 \%, 19.4 \%$, and $23.4 \%$ respectively (refer Table 2). This shows that a significant amount of occupancy variation can be occur between days. One noticable difference between the simulations is the absence of noise in the ABM simulations. Since the ABM requires agents to follow a predefined path, this prevents people pacing between areas. In this respect, the ABM seems to capture the subtle occupancy variablility better than MVGM.

While both models produce plausible simulations, each has its advantages and disadvantages. The ABM can be ap- plied to other structures where an occupancy sensing infrastructure does not exist by training the $\mathrm{ABM}$ with data from another building with similar dynamics. This is not possible with the MVGM. The ABM is useful for building designers looking to maximize energy savings. The MVGM is more useful for real time prediction. Unlike the ABM which simulates occupancies offline, the MVGM can predict occupancy given the time of day and current occupancy by calculating the posterior probability given the current occupancy on all or part of the building.

The results that can drawn are limited by the data available. Though it would be preferable to gather data beyond 48 hours to capture weekly, monthly, and quarterly trends, the labor required to process ground truth data makes gathering additional data impractical. Ideally, less accurate data can be automatically gathered by SCOPES and used for model development. However, currently the SCOPES cameras cannot be placed in several key transition boundaries in the hallway because of physical restrictions and University regulations. We are working toward overcoming these restrictions with the eventual goal of collecting longer traces of data from SCOPES to model short and long term trends.

\section{Optimal L-HVAC Strategies}

Traditionally, lighting, temperature, and ventilation (outside air - OA) control are based on schedules that rely on room usage assumptions. However, this assumption frequently overestimates the occupancy of spaces. On the other hand, if occupancy information is known, one could control lights, temperature, and ventilation levels to the appropriate level required, and set them back to minimum or off conditions when no occupancy is detected, resulting in energy savings. In this paper we illustrate the impact of ventilation control strategy on energy savings. Ventilation air is introduced into the building to avoid poor indoor air quality and is a function of the number of people in the building and the square footage; simply knowing if a room is occupied is not sufficient for venting. In absence of real-time occupancy information, OA amount is fixed based on maximum design occu- 
pancy assumption. Excess outdoor air has significant penalty on heating energy and depending on the outside temperature, even cooling energy. Thus varying the amount of air during nonfree cooling times to the minimum required to maintain acceptable indoor air quality based on actual real time occupancy has potential for saving energy.

An existing eQuest model [3] of Classroom and Office building (COB) on the UC Merced campus, developed during the building design stage, was used to understand the energy savings potential of using occupancy estimation for ventilation control. EQuest is an hourly whole building energy simulation tool used widely by the building community. It uses information on building configuration, schedules of building usage including people, lighting, plug loads, HVAC system configuration, expected cooling, heating and ventilation levels, and weather conditions to calculate the building energy consumption.

Two ventilation control strategies were simulated: base and new. In the base case, the OA quantity is based on maximum design occupancy, available during the occupied time ( 8 am $10 \mathrm{pm})$. This quantity is fixed during the occupied time, irrespective of occupancy. The new ventilation strategy has OA quantities in all zones following the occupancy in the respective zones. All the strategies simulated consider the applicable codes. The daily actual occupancy levels over time for various HVAC zones in COB is estimated from available information on schedules for classrooms and an ABM for people movement in offices.

\subsection{Occupancy schedule for part of $\mathrm{COB}$}

The S\&E building has multitude of HVAC systems deployed for a variety of purposes including office spaces as well as clean room or laboratories. Due to the simplicity of the HVAC configuration in the COB building, and its primary usage for offices and classrooms with variable occupancies, the COB building was chosen for the energy savings estimation. Also, since energy models had previously been developed for the COB building, the goal was to take advantage of these models and examine potential energy savings for this building using simulated occupancy schdedules. Currently it is not possible to measure occupancies directly in the COB building. However, since the S\&E building has similar traffic patterns to the COB, an ABM trained using data from the $\mathrm{S} \& \mathrm{E}$ building was developed to construct occupancies schedules for some portions of the COB building.

The occupancy modeling work for the UC Merced COB has been focused on the office section of second floor. Statistical distributions of parameters which define occupant itinerary; arrival time, dwell time, and number of stops, are extracted from the ABM from the S\&E building, which is expected to have similar traffic. The information regarding different types of occupants that use the building (and their relative proportions) and occupancy level for whole office section, is derived from different types of rooms in the section and maximum occupancy expected in each room. Finally, by Monte Carlo sampling of the occupant itinerary parameters and averaging over different realizations of traffic patterns obtained from simulating the model, occupancy profile over the course of the day for each room is generated. This helps us assess the impact of using the time resolved occupancy distribution on energy savings.

\subsection{Potential Energy Savings}

Using simulation data from the COB ABM, we find that $5 \%$ of HVAC energy savings is possible compared to the current outside ventilation air control strategy and about $14 \%$ HVAC energy savings is possible when compared with base-case outside air control strategy. Sensitivity calculations of energy savings to occupancy estimation errors and sensor bias were conducted. Results show that $20 \%$ occupancy estimation errors have negligible impact $(0.28 \%)$ on HVAC energy savings estimation of $14 \%$. A sensor bias of $20 \%$ results in $3 \%$ change in energy savings estimation.

\section{Conclusions}

In this work, we present the first steps in understanding dynamic occupancy levels and patterns in buildings, means by which they can be estimated, and the energy efficiency gains possible by utilizing actual facility usage information for building controls. As proof of concept, we deployed an 16-node wireless camera sensor network in a multi-function building to determine the occupancy resolution one can conceive of obtaining in a typical building. These results in turn were used to demonstrate an example of an occupancy-based energy control. The results suggest that, in many buildings and for many building uses, we will find that knowing the occupancy and usage patterns will result in significantly higher energy savings compared to strategies assuming fixed occupancy and usage patterns. Our future research directions involve doing online L-HVAC control using only a wireless camera sensor network to provide occupancy estimates for longer durations (days, months).

\section{Acknowledgments}

This work was supported in part by the Department of Energy and the California Energy Commission, and performed under U.S. Department of Energy Contract No. DE-AC0205CH11231 and the California Institute for Energy and the Environment under contract No. MUC-09-03.

\section{References}

[1] G. A. Bierlaire, M and M. Webers. Behavioral dynamics for pedestrians. In 10th International Conference on Travel Behavior Research, Aug. 2003.

[2] M. J. Brandemuehl and J. E. Braun. The impact of demand-controlled and economizer ventilation strategies on energy use in buildings. ASHRAE Transactions, 105(2), 1999

[3] eQuest Building Energy Analysis Tool. http://www.doe2.com/.

[4] A. Kamthe, L. Jiang, M. Dudys, and A. Cerpa. SCOPES: Smart Cameras Object Position Estimation System. In Proceedings of the 6th European Conference on Wireless Sensor Networks(EWSN 2009), pages 279-295, Cork, Ireland, Feb. 2009. Springer-Verlag.

[5] F. W. Payne. Energy management control system handbook. Fairmont Press, 1984

[6] S. P. Meyn, A. Surana, Y. Lin, S. M. Oggianu, S. Narayanan, T. Frewen. A sensor-utility-network method for estimation of occupancy distribution in buildings. In Proceedings of IEEE Conference on Decision and Control, Shanghai, China, 2009.

[7] A. Schadschneider. Pedestrian and Evacuation Dynamics, chapter Cellular automaton approach to pedestrian dynamics theory, pages 75-86. Springer, 2002. 